\title{
Graphene-based metasurface absorber for the active and broadband manipulation of terahertz radiation
}

\author{
EkIn Bircan Boşdurmaz, ${ }^{1,2}$ (i) Hodjat HajıAN, ${ }^{1, *}$ (i) Veysel ERÇAĞLAR, ${ }^{1,2}$ (i) AND \\ EKMEL ÖZBAY 1,2,3,4 \\ ${ }^{1}$ NANOTAM-Nanotechnology Research Center, Bilkent University, Ankara 06800, Turkey \\ ${ }^{2}$ Department of Electrical and Electronics Engineering, Bilkent University, Ankara 06800, Turkey \\ ${ }^{3}$ Department of Physics and UNAM-Institute of Materials Science and Nanotechnology, Bilkent University, 06800 Ankara, Turkey \\ ${ }^{4}$ e-mail: ozbay@bilkent.edu.tr \\ *Corresponding author: hodjat.hajian@bilkent.edu.tr
}

Received 14 April 2021; revised 27 June 2021; accepted 8 July 2021; posted 8 July 2021 (Doc. ID 427975); published 10 August 2021

\begin{abstract}
Graphene-based metasurface nearly perfect absorbers (MPAs) can be used as an efficient tool for the active control and manipulation of waves in the terahertz $(\mathrm{THz})$ gap. Here, we propose a novel, to the best of our knowledge, graphene-based MPA that is designed based on a simple configuration and is capable of absorbing THz radiation within a broad bandwidth of almost $3 \mathrm{THz}$ with polarization-insensitive and omnidirectional characteristics. The MPA comprises a periodic array of graphene patches with two different dimensions that are separated from a gold bottom reflector with an $\mathrm{SiO}_{2}$ spacer layer. The broadband spectral response of the MPA, which is also verified by analytical calculations, is due to the support of propagating surface plasmon excitations and can be either actively tuned via changes in the chemical potential of graphene or passively adjusted by the modification of the geometrical parameters of the patches and thickness of the spacer layer. As a complement to the previous studies in the literature, due to the simplicity of its design and broad spectral response, it is believed that the suggested graphene-based MPA will find potential applications in THz spectroscopy and communications. (๑) 2021 Optical Society of America
\end{abstract}

https://doi.org/10.1364/JOSAB.427975

\section{INTRODUCTION}

Metasurfaces, two-dimensional (2D) metamaterials composed of subwavelength resonators, can be utilized to efficiently tailor an incident electromagnetic wave $[1,2]$. They have been used in many applications, such as flat lenses [3,4], metamirrors [5,6], polarization converters $[7,8]$, full-color printers [9], holographs $[10,11]$, optical vortex generators $[12,13]$, and beam splitters $[14,15]$. Light absorption is another eye-catching characteristic of these artificial systems [16], and metastructures with a nearly perfect light absorption characteristic are referred to as metasurface/metamaterial nearly perfect absorbers (MPAs). In order to realize nearly perfect light absorption, reflectance is suppressed by matching the effective impedance of the metasurface to that of the incident medium [17]. Simultaneously, transmittance may be eliminated by introducing another metallic film acting as a mirror or by using a similar mechanism in a multilayer system [18]. Based on these approaches, metasurface nearly perfect light absorbers can be categorized as narrowband MPAs [19-25] and broadband MPAs [26-41]. They can also be classified according to their wavelength of operation in the visible [26-29,42,43], infrared [30,44,45], terahertz $(\mathrm{THz})$ [21-25,31-40], and microwave ranges [19,20,41].
The $\mathrm{THz}$ spectrum is referred to as electromagnetic waves ranging from 0.1 to $10 \mathrm{THz}$ that bridges infrared light and microwaves. This range of frequency, which is also called the $\mathrm{THz}$ gap, has many important characteristics. Due to their low energy, $\mathrm{THz}$ radiations cannot interact with the electrons in atoms. In other words, they do not have the potential to ionize materials, and therefore they cannot damage living tissues. This characteristic makes $\mathrm{THz}$ waves very practical for medical imaging and biomedical applications [46]. Moreover, many materials that are opaque to visible light are transparent to $\mathrm{THz}$ radiation. This feature makes $\mathrm{THz}$ waves practical for scanning passengers in airports [47]. Furthermore, the $\mathrm{THz}$ frequencies offer a wide bandwidth in an uncrowded part of the electromagnetic spectrum. Therefore, they can be employed for achieving high-rate data transfer in wireless technology [48]. In view of the useful potential applications of $\mathrm{THz}$ radiation, progress has been made in $\mathrm{THz}$ generation and detection (see, e.g., $[49,50]$ ) as well as in achieving functional metasurfaces for lensing, polarization conversion, splitting, and other applications (see, e.g., $[5,7,14])$. Employing gold-based metasurfaces integrated with semiconductors as active elements has also been introduced as an efficient method to real-time control and to manipulate 
$\mathrm{THz}$ radiation [51]. Vanadium dioxide $\left(\mathrm{VO}_{2}\right.$, which exhibits an insulator-to-metal transition and 3 orders of magnitude increase in $\mathrm{THz}$ electrical conductivity, has also been utilized in the realization of active $\mathrm{THz}$ metasurfaces [52]. Metasurfaces based on graphene-that is, a low-loss plasmonic material with gate-tunable characteristics-are also the most notable category of active devices for the efficient steering of $\mathrm{THz}$ waves [53]. Considering the importance of light absorbers in $\mathrm{THz}$ spectroscopy and communications, on the one hand, and achieving metasurfaces for the active manipulation of $\mathrm{THz}$ waves, on the other hand, have recently led researchers to the design and realization of graphene-based metasurfaces for broadband $\mathrm{THz}$ light absorption. The first approach for the realization of graphene-based broadband MPAs is to utilize cascaded metasurfaces that are composed of multilayers of patterned graphene surfaces (see, e.g., [38-40]). However, the fabrication and gating of the MPAs in this approach are complex, thereby making it less effective. The second and more effective methodology for the realization of broadband MPAs is to employ appropriately designed metasurfaces based on a single layer of graphene [31-36]. Nevertheless, the reported absorption bandwidths of these metasurfaces are less than $1.5 \mathrm{THz}$. Recently, a broadband graphene-based MPA has been reported with a bandwidth of almost $3 \mathrm{THz}$ [37], but the patterned graphene sheet is too complex and may not be realized in practice, and that makes the functionality of the suggested device less efficient. Consequently, achieving alternative graphene-based MPAs with doable and less complex designs is still appreciated.

In the present paper, we design and propose a novel graphenebased MPA with broadband, polarization-insensitive, and omnidirectional characteristics. The graphene-based MPA comprises a single layer of graphene square patches with two different dimensions that are separated from an optically thick $\mathrm{Au}$ bottom reflector with an $\mathrm{SiO}_{2}$ spacer layer. The suggested MPA is capable of absorbing $\mathrm{THz}$ radiation within a bandwidth of almost $3 \mathrm{THz}$, for which the numerically obtained absorption response of the MPA is verified by analytical calculations. The spectral location of the broad bandwidth can be either actively tuned by modifying the chemical potential of graphene or passively amended by changing the geometrical parameters.
Moreover, owing to the simplicity of its design, the proposed MPA can be realized in practice. It is expected that this simple and novel design will find potential applications in $\mathrm{THz}$ spectroscopy and communications.

\section{ANALYTICAL MODELING}

In this section, we provide an analytical approach for the investigation of the absorption of the graphene-based MPA based on an equivalent circuit model. Using this approach, we can evaluate and analyze our numerical results (see Section 3) that are obtained by finite-difference time-domain (FDTD) simulations [54].

The perspective and top-view schematics of a unit cell of the graphene-based MPA are illustrated in panels (a) and (b) of Fig. 1. As seen from these panels, each unit cell with periodicity $p_{x}=p_{y}$ comprises an array of square patches of widths $w_{1}$ and $w_{2}$ [with gaps $d$ between them in a way that $\left.p_{x}=p_{y}=3\left(\frac{w_{1}+w_{2}}{2}+d\right)\right]$ and separated from an optically thick Au mirror by an $\mathrm{SiO}_{2}$ spacer layer of width $t_{s}$. According to the configuration of the patches, the equivalent circuit model of the proposed MPA is presented in Fig. 1(c). In this model, each graphene patch is described by an impedance as [23]

$$
Z_{1,2}=\left(\frac{p_{x}}{3 w_{1,2}}\right)^{2}\left(\frac{1}{\sigma_{g}}-\frac{n_{1,2}}{i \omega \varepsilon_{\text {eff }}}\right) / m_{1,2} .
$$

Here $n$ and $m$ are fitting parameters, $\varepsilon_{\text {eff }}=\varepsilon_{0}\left(1+n_{\mathrm{SiO}_{2}}^{2}\right) / 2$, and $\sigma_{g}=\sigma_{g}^{\text {intra }}+\sigma_{g}^{\text {inter }}$ is the optical conductivity of graphene, which is a function of frequency and temperature and can be formulated as [55]

$$
\begin{aligned}
\sigma_{g}^{\text {intra }}(\omega, T)= & \frac{e^{2}}{4 \hbar} \frac{i}{2 \pi}\left\{\frac{16 k_{B} T}{\hbar \Omega} \ln \left(2 \cosh \left(\frac{\mu}{2 k_{B} T}\right)\right)\right\}, \\
\sigma_{g}^{\text {inter }}(\omega, T)= & \frac{e^{2}}{4 \hbar}\left\{\frac{1}{2}+\frac{1}{\pi} \arctan \left(\frac{\hbar \Omega-2 \mu}{2 k_{B} T}\right)\right. \\
& \left.-\frac{i}{2 \pi} \ln \frac{(\hbar \Omega+2 \mu)^{2}}{(\hbar \Omega-2 \mu)^{2}+\left(2 k_{B} T\right)^{2}}\right\},
\end{aligned}
$$

(a)

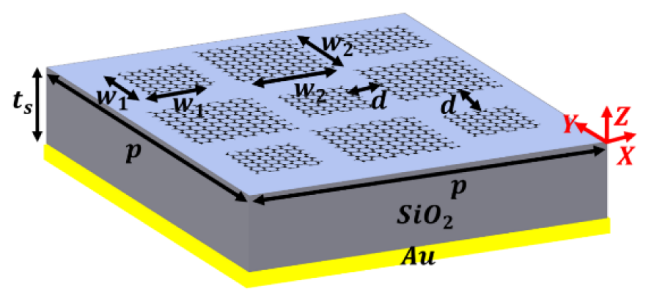

(b)

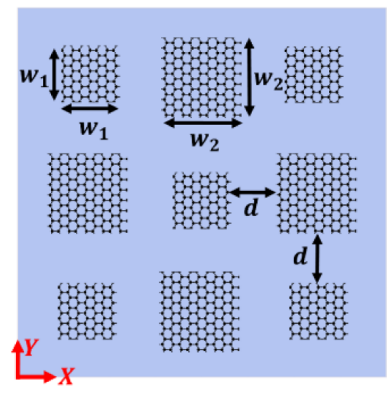

(c)

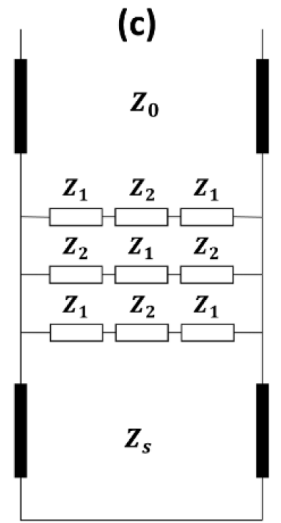

Fig. 1. (a) and (b) Perspective and top-view schematics of the unit cell of the graphene-based MPA, respectively. As shown, in this arrangement, the unit cell comprises an array of two graphene patches of widths $w_{1}$ and $w_{2}$ that are detached by a gap of width $d$, and $p_{x}=p_{y}=3\left(\frac{w_{1}+w_{2}}{2}+d\right)$. As seen, the top graphene patches are separated from the optically thick Au mirror by an $\mathrm{SiO}_{2}$ spacing layer with the thickness $t_{s}$. (c) Equivalent circuit model for the proposed absorber. In this model, $Z_{1}$ and $Z_{2}$ stand for the impedances of $w_{1}$ and $w_{2}$ graphene patches, and $Z_{0}$ and $Z_{1}$ refer to the impedances of air and the spacing layer, respectively. 
with $\Omega=\omega+i \tau^{-1}, e$ is the electron charge, $k_{B}$ is the Boltzmann constant, $\tau$ is the electron relation time, and $\hbar$ is the Plank constant over $2 \pi$. Consequently, the total effective impedance of the top patches is written as

$$
Z_{\text {eff }}=\frac{\left(Z_{1}+Z_{2} / 2\right)\left(Z_{1}+2 Z_{2}\right)}{2 Z_{1}+5 Z_{2} / 2} .
$$

As a result, the input impedance can be calculated as

$$
Z_{\text {in }}=\frac{Z_{\text {eff }} Z_{s}}{i \cot \left(\frac{n_{s \omega}}{c} t_{s}\right) Z_{\text {eff }}+Z_{s}}
$$

and the reflection $(R)$ of light from the MPA is given by

$$
R=\left|\left(Z_{\text {in }}-Z_{0}\right) /\left(Z_{\text {in }}+Z_{0}\right)\right|^{2} .
$$

Here $c$ is the speed of light, $Z_{s}=Z_{0} / n_{s}, n_{s}=n_{\mathrm{SiO}_{2}}$, and $Z_{0}$ is the free-space impedance. Note that, due to the presence of the optically thick bottom gold layer, light transmission through the metasurface is zero, and therefore the absorption of light is calculated as $A=1-R$. In Figs. 3(a) and 3(b), we compare the analytical results calculated from Eqs. (4) and (5) with the ones obtained numerically. Note that, in our calculations in this paper, $\tau=0.1 \mathrm{ps}, w_{1}=3 \mu \mathrm{m}, w_{2}=4 \mu \mathrm{m}$, and the refractive index data of $\mathrm{SiO}_{2}$ is chosen according to Ref. [56]. It is noteworthy that, in the $\mathrm{THz}$ range, the optical conductivity of a multilayer graphene that is composed of $N_{g}$ layers can be expressed as $\frac{N_{g} i e^{2} \mu}{\pi \hbar^{2}\left(\omega+i \tau^{-1}\right)}$. Consequently, at the same frequency and with an equal value of $\tau$, graphene-based metasurfaces with identical values of $N_{g} \mu$ are expected to exhibit similar optical responses. In other words, a metasurface based on a single layer graphene with $\mu=1 \mathrm{eV}$ acts as a bilayer graphene-based metasurface with $\mu=0.5 \mathrm{eV}$.

\section{RESULTS AND DISCUSSIONS}

In Figs. 2(a) and 2(b), we provide the numerically obtained results for the broadband graphene-based MPA and compared them with the analytical calculations.

For the MPA shown in Figs. 1 (a) and 1(b), at $\mu=1 \mathrm{eV}$, the analytically calculated input impedance that is based on Eq. (5) for $n_{1,2}=(0.377,0.446)$ and $m_{1,2}=(2.721,0.418)$ is compared to the numerically obtained one in Fig. 2(a). As seen, there is an acceptable agreement between the real and imaginary parts of the analytical and numerical impedances. This agreement leads to the match between the analytically calculated absorption spectrum based on Eq. (6) [dotted blue] and the numerically calculated one shown in the solid black curve in Fig. 2(b). According to this figure, the broadband absorption region extends from $f=3.64 \mathrm{THz}$ to $f=6.55 \mathrm{THz}$; i.e., an almost $3 \mathrm{THz}$ absorption bandwidth is obtained. According to Palik's data [57], $\mathrm{SiO}_{2}$ is a lowly dispersive material in the $\mathrm{THz}$ region, where $1.96<\operatorname{Re}\left(n_{s}\right)<2.2$ and $0.01<\operatorname{Im}\left(n_{s}\right)<0.065$. Taking $n_{s}=2$ as a hypothetical material for the spacer layer for which the refractive index is close to $n_{\mathrm{SiO}_{2}}$, it is observed that the broadband absorption region is blueshifted and slightly narrowed, as the dotted
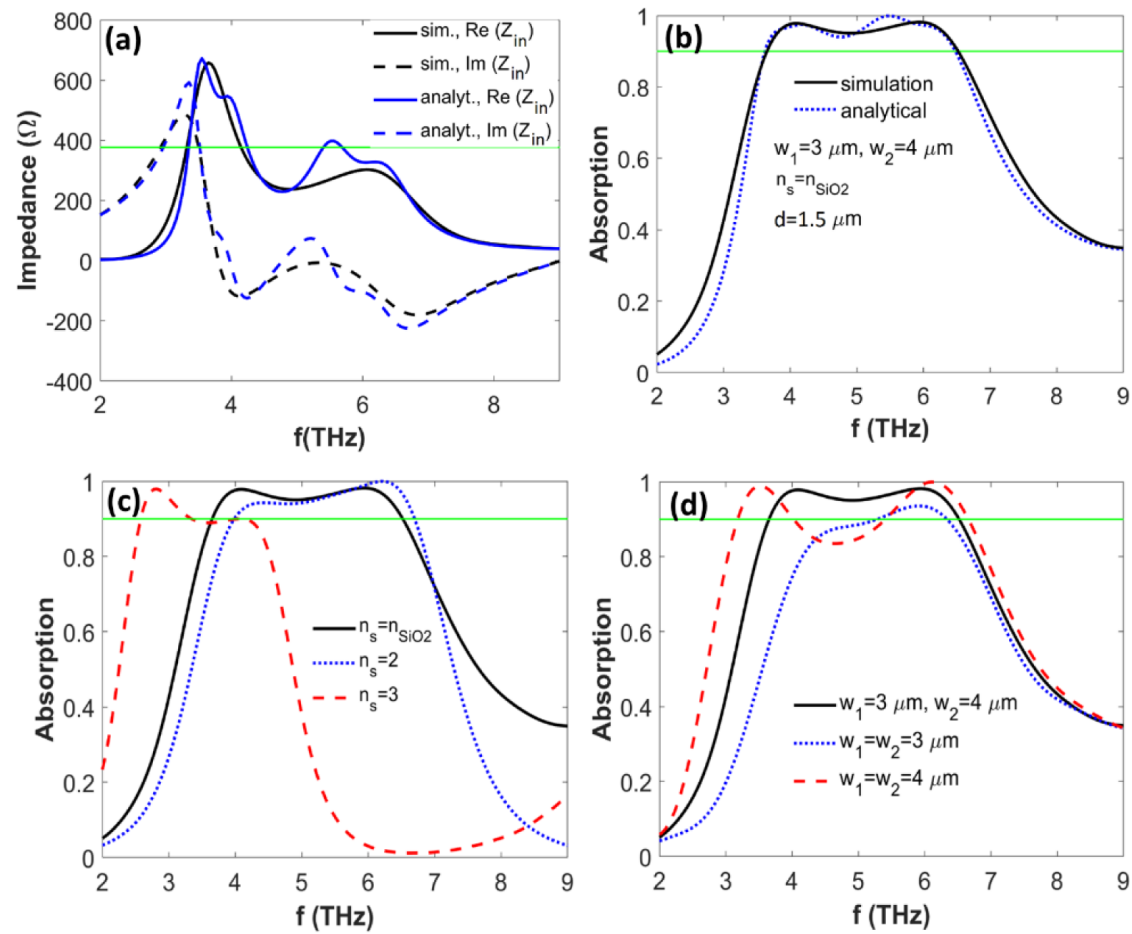

Fig. 2. (a) Analytically (blue curves) and numerically (black curves) obtained input impedances of the graphene-based MPA. Solid green curve indicates $Z_{0}=120 \pi$. (b) Dotted blue and solid black curves illustrate the analytically and numerically obtained absorption of the MPA for normal incident light, respectively. The optimized values of the geometrical parameters ( $d$ and $t_{s}$ ) are also highlighted in this panel. (c) Absorption of the MPA for the different values of $n_{s}$, while the geometrical parameters are kept as in panel (b). In panel (d), absorption of the MPA (solid black curve) is compared to the cases for which the widths of the patches are equal as $3 \mu \mathrm{m}$ (dotted blue curve) and $4 \mu \mathrm{m}$ (dashed red curve). Note that, in panels (b)-(d), the solid green line indicates $A=90 \%$ and for all of the results illustrated in this figure $\mu=1 \mathrm{eV}$. The solid black curves in these panels are the same. 

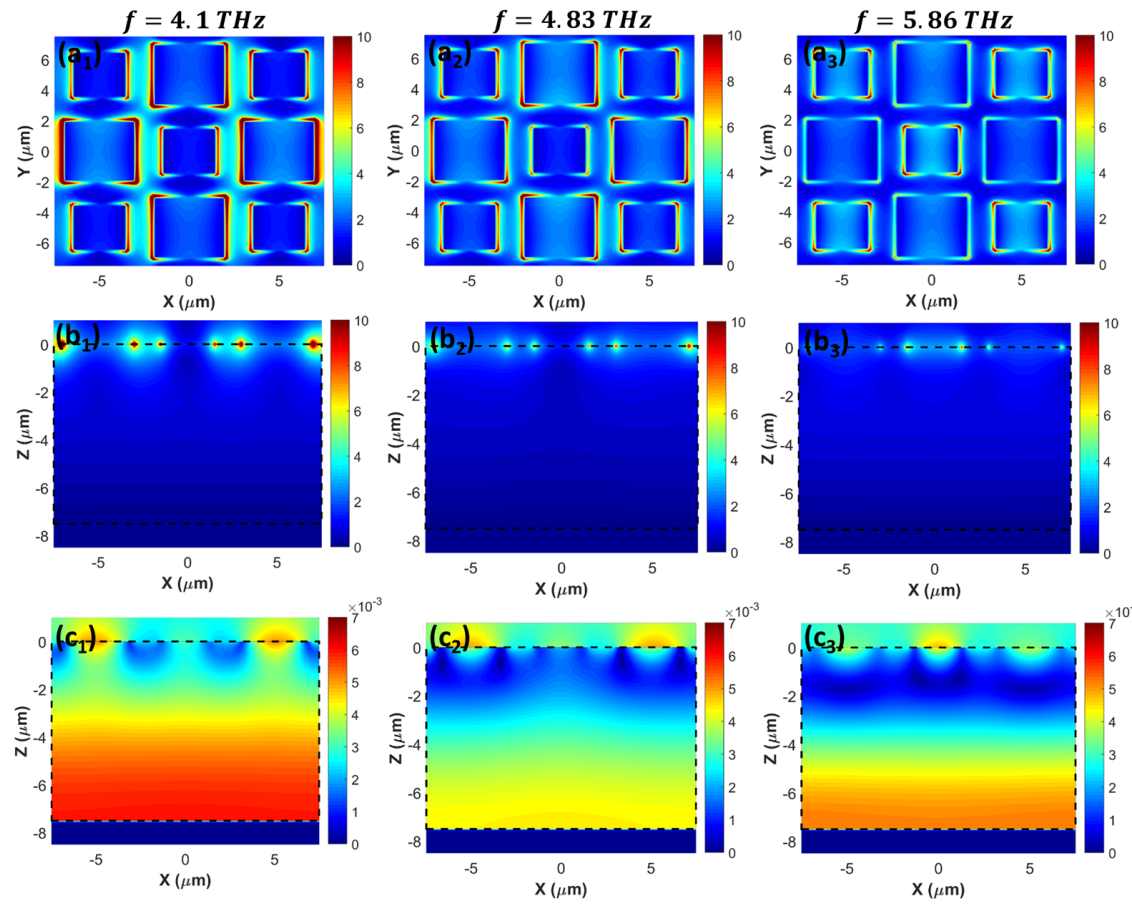

Fig. 3. Panels $\left(\mathrm{a}_{i}\right),\left(\mathrm{b}_{i}\right)$, and $\left(\mathrm{c}_{i}\right)[i=1,2,3]$, respectively, illustrate normalized values of the top view of $|\boldsymbol{E}|$, side view of $|\boldsymbol{E}|$, and side view of $|\boldsymbol{H}|$ at the two peaks $(f=4.1 \mathrm{THz}$ and $5.86 \mathrm{THz})$ and the deep $(f=4.83 \mathrm{THz})$ in the nearly perfect and broadband absorption bandwidth of the solid black curve of Fig. 2(a). The dashed rectangle in panels $\left(b_{i}\right)$, and $\left(c_{i}\right)$ indicates the $\mathrm{SiO}_{2}$ spacer layer.

blue and solid black curves in Fig. 2(c) are compared. On the other hand, the dashed red curve in this figure proves that by increasing the refractive index of the spacer layer to a larger hypothetical value (i.e., $n_{s}=3$ ), the broadband response of the MPA is totally distorted and a narrowband response at lower frequencies is achieved. It is also observed from Fig. 2(d) that once all the patches of the MPA have the same dimensions with $w_{1}=w_{2}=3 \mu \mathrm{m}$ (dotted blue curve) or $w_{1}=w_{2}=4 \mu \mathrm{m}$ (dashed red curve), the broadband absorption response of the MPA is transferred to a narrowband one with a single resonance or one with two resonances, respectively.

To understand the physical mechanism behind the broadband response of the proposed MPA, in Fig. 3, we examine the electric and magnetic field profiles at the two peaks $(f=4.1 \mathrm{THz}$ and $5.86 \mathrm{THz})$ and the deep $(f=4.83 \mathrm{THz})$ in the nearly perfect and broadband absorption region of the solid black curve of Fig. 2(b).

The top-view $|\boldsymbol{E}|$ mode profiles of the resonant peaks of absorption at $f=4.1 \mathrm{THz}$ and $5.86 \mathrm{THz}$ are shown in panels $\left(a_{1}\right)$ and $\left(a_{3}\right)$ of Fig. 3. It is seen from these panels that, at $f=4.1 \mathrm{THz}$, the larger graphene patches $\left(w_{2}\right.$ patches) are strongly excited, while at $5.86 \mathrm{THz}$, the smaller patches ( $w_{1}$ patches) are mostly excited. On the other hand, at $f=4.83 \mathrm{THz}$, that corresponds to the deep in the broadband absorption region of Fig. 2(b), in which both $w_{1}$ and $w_{2}$ graphene patches are almost equally responsible for the plasmonic excitation of the MPA as seen in Fig. 3(a2). In agreement with the top-view mode profiles, the side-view of $|\boldsymbol{E}|$, which is depicted in Figs. 3(b1 )-3(b3), shows that those plasmonic excitations are strongly bound to the graphene patches. This is in agreement with the highly localized $\mathrm{THz}$ surface plasmon polaritons supported by a sheet of graphene. From the side view of $|\boldsymbol{H}|$ at those frequencies, it is obvious that the magnetic field is not only strongly enhanced between the graphene patches, but it also has a strong interaction with the bottom Au mirror and is confined beneath the patches in the spacer layer as seen in Figs. 3(c1)-3(c3). This observation reveals that the propagating surface plasmon resonances supported by the graphene-based MPA are responsible for the broadband absorption response. It should be noted that changes in the thickness of the spacer layer considerably affect the nearly perfect absorption response of the MPA (see Fig. 4).

Taking $d=1.5 \mu \mathrm{m}$ and $\mu=1 \mathrm{eV}$ in the calculations, the absorption spectrum of the MPA for the different values of the thickness of the spacing $\mathrm{SiO}_{2}$ layer are shown in Fig. 4(a). In agreement with this figure, Fig. 4(b) represents the absorption of the MPA for three values of the thickness of the spacer layer; i.e., $t_{s}=4 \mu \mathrm{m}$ (dotted blue curve), $t_{s}=7.5 \mu \mathrm{m}$ (solid black curve), and $t_{s}=10 \mu \mathrm{m}$ (dashed red curve). It is clear from Fig. 4 that changes in the thickness of the spacer layer significantly modify the absorption response. In other words, at the optimized value of $t_{s}$ (i.e., $t_{s}=7.5 \mu \mathrm{m}$ ), the broadband response is observed, while by decreasing/increasing this thickness, it is possible to achieve two/one distinct narrow resonance(s). For example, as seen from the dotted blue curve in Fig. 4(b), two strong absorptive resonances are achieved at $f=4.53 \mathrm{THz}$ and $f=9.82 \mathrm{THz}$.

One of the practically important characteristics of the MPAs is the dependency of their absorption response to the polarization and angle of the incident light. This feature is examined in Fig. 5. 

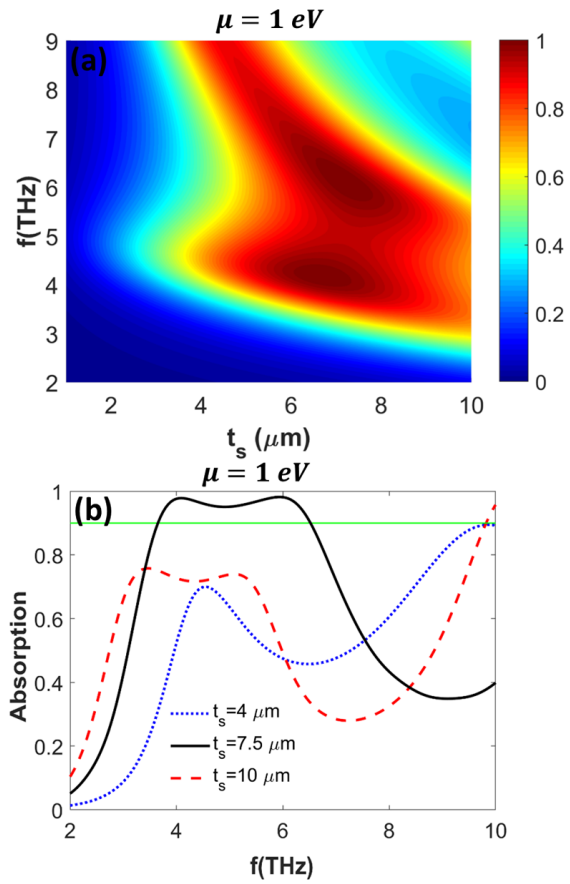

Fig. 4. (a) Absorption of the graphene-based MPA as a function of the thickness of the spacer layer $\left(t_{s}\right)$. (b) Similar to panel (a), while the absorption values are depicted for the three values of $t_{s}$, i.e., $t_{s}=4 \mu \mathrm{m}$ (dotted blue curve), $t_{s}=7.5 \mu \mathrm{m}$ (solid black curve; the optimized result discussed in Fig. 2 ), and $t_{s}=10 \mu \mathrm{m}$ (dashed red curve). Note that, here, the results are obtained for the design shown in Fig. 1(a) with $d=1.5 \mu \mathrm{m}$ and $\mu=1 \mathrm{eV}$.

Figure 5(a) illustrates the dependency of the absorption spectra of the MPA for the $x$-polarized or TM $(\phi=0)$ and $y$-polarized or $\operatorname{TE}(\phi=\pi / 2)$ incident light. Here, the azimuthal angle $\phi$ denotes the in-plane direction of the electric field with respect to the $x$ and $y$ axes. It is seen from this figure that the nearly perfect absorptive response of the graphene-based MPA is kept unchanged up to $\theta=50^{\circ}$ while the bandwidth of the spectrum is shrunk for larger incident angles. For the case that the azimuthal angle is chosen as $\phi=45^{\circ}$, the absorption response of the MPA also remains as broad as the normal incidence illumination up to $\theta=45^{\circ}$, but for the larger angles of incidence, two narrowband perfect absorption peaks are achieved as shown in Fig. 5(b). Consequently, the designed graphene-based metasurface can act as a broadband, polarization-insensitive, and omnidirectional MPA up to $=45^{\circ}$, thereby meeting the needs for practical applications.

Taking $d=1.5 \mu \mathrm{m}$ and $\mu=1 \mathrm{eV}$, we have designed a graphene-based MPA to achieve a THz broadband absorption response with the absorption bandwidth of almost $3 \mathrm{THz}$. In Fig. 6, we examine how the absorptive response of the MPA can be actively tuned via changes in the chemical potential of graphene.

It is seen in Fig. 6(a) that, for $d=1.5 \mu \mathrm{m}$, the MPA response can be noticeably tuned from a narrowband absorption at low values of the chemical potential of graphene [e.g., $A=64 \%$ at $f=5.26 \mathrm{THz}$ and $\mu=0.2 \mathrm{eV}$; see the dashed red curve in Fig. 6(b)] to a broadband one at $\mu>0.60 \mathrm{eV}$. Moreover, the broadband absorption bandwidth is also blueshifted and widened by increasing the chemical potential of graphene, as

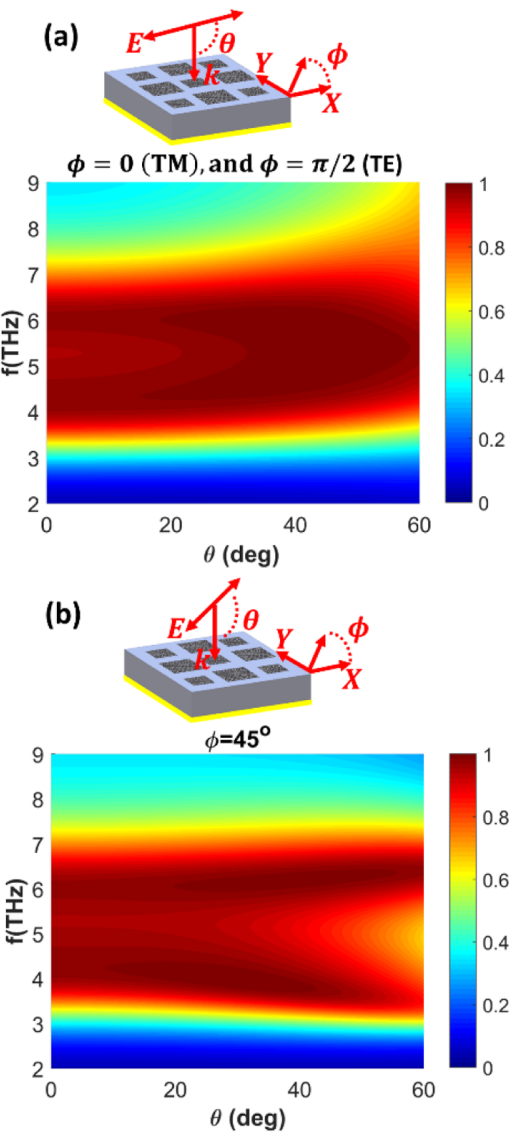

Fig. 5. Dependence of the absorption spectra of the MPA on the angle of incidence $\theta$ for three different values of the azimuthal angle $\phi$; i.e., (a) $\phi=0, \pi / 2$ and (b) $\phi=45^{\circ}$.
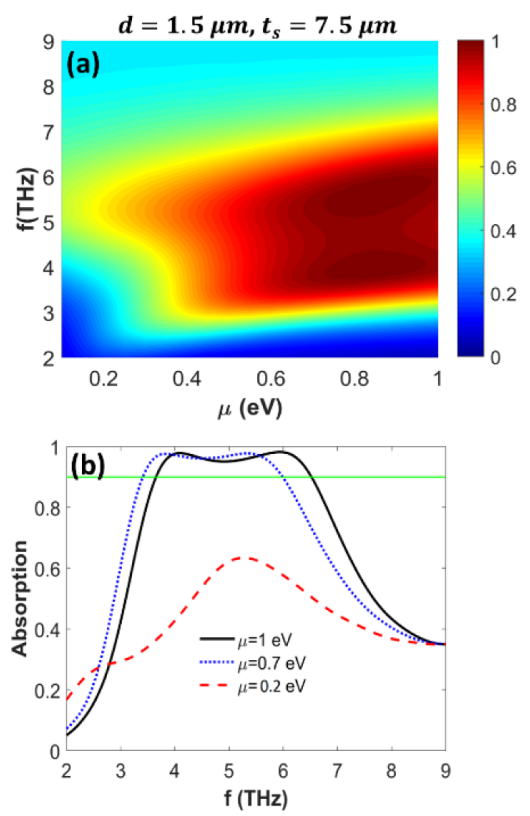

Fig. 6. (a) Absorption spectra of the MPA as a function of the chemical potential of graphene for $d=1.5 \mu \mathrm{m}$ and $t_{s}=7.5 \mu \mathrm{m}$. In agreement with panel (a), (b) illustrates the absorption response for $\mu=1 \mathrm{eV}$ (solid black curve), $\mu=0.7 \mathrm{eV}$ (dotted blue curve), and $\mu=0.5 \mathrm{eV}$ (dotted red curve). The solid green line indicates $A=0.9$ and the results are obtained at normal incidence. 

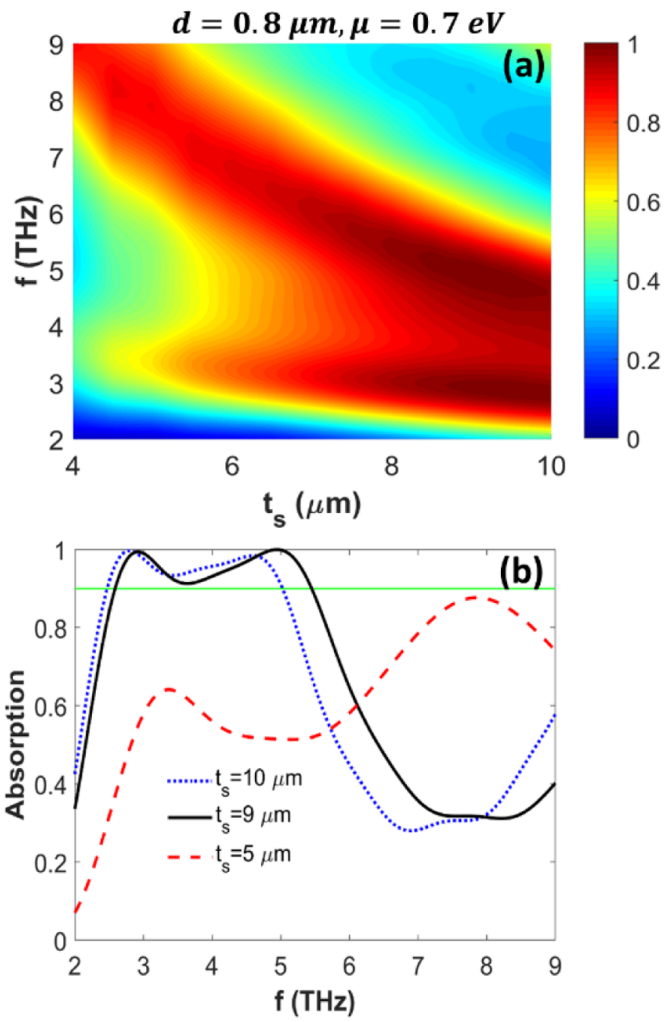

Fig. 7. (a) illustrates the dependency of the MPA's absorption spectrum for $d=0.8 \mu \mathrm{m}$ and $\mu=0.7 \mathrm{eV}$ on the spacer layer thickness. (b) Results of panel (a) for three different values of $t_{s}$, i.e., $t_{s}=7.5 \mu \mathrm{m}$ (dotted blue curve), $t_{s}=9 \mu \mathrm{m}$ (solid black curve), and $t_{s}=5 \mu \mathrm{m}$ (dashed red curve). The results are obtained at normal incidence and the solid green line highlights $A=0.9$. Note that, for this design, the locations of the $w_{1}$ and $w_{2}$ patches that are shown in Fig. 1(a) are interchanged, i.e., there are five (four) $w_{2}\left(w_{1}\right)$ patches in the unit cell.

the dotted blue cure $(\mu=0.7 \mathrm{eV})$ in Fig. 5(b) is compared to the solid black one $(\mu=1 \mathrm{eV})$. In other words, the spectral tuning of the bandwidth is achieved. Note that the bandwidth for the $\mu=0.7 \mathrm{eV}$ case in Fig. $5(\mathrm{~b})$ is $2.6 \mathrm{THz}$ (located at 3.4 to $6 \mathrm{THz})$.

However, from a practical point of view, obtaining a broadband absorption with $3 \mathrm{THz}$ bandwidth may be more desirable for $\mu<1 \mathrm{eV}$, e.g., obtaining $0.7 \mathrm{eV}$ may be more feasible in practice for the chemical potential of graphene. To this end, taking $\mu=0.7 \mathrm{eV}$ and decreasing $d$ from 1.5 to $0.8 \mu \mathrm{m}$, the absorption spectrum of the MPA as a function of the thickness of the $\mathrm{SiO}_{2}$ spacer layer is examined in Fig. 7(a). It is clearly observed in this figure that for smaller values of $t_{s}$, the metasurface exhibits narrowband responses, e.g., for $t_{s}=5 \mu \mathrm{m}$, two strong absorption resonances $(A>60 \%)$ are obtained at $f=3.35 \mathrm{THz}$ and $f=7.86 \mathrm{THz}$ as seen from the dashed red curve in Fig. $7(\mathrm{~b})$. On the other hand, by increasing $t_{s}$, the graphene-based metasurface acts as a broadband MPA and represents a bandwidth of almost $3 \mathrm{THz}$ at $t_{s}=9 \mu \mathrm{m}$ for the case of $\mu=0.7 \mathrm{eV}$ as seen from the solid black curve in Fig. $7(\mathrm{~d})$. Note that, in this study, we have introduced two designs with broadband responses (bandwidth $\sim 3 \mathrm{THz}$ ) in Fig. 2 and Fig. 7. The results of Fig. 2 are obtained at $\mu=1 \mathrm{eV}$, while for the results of Fig. 3, the value of the chemical potential of graphene is taken as $\mu=0.7 \mathrm{eV}$. From a practical point of view, $\mu=0.7 \mathrm{eV}$ is expected to be achieved by top-gating in the experiment (see Ref. [57]). However, as we explained in Section 2 and according to FDTD simulations, since $N_{g} \mu$ affects the response of our designs, the results of Figs. 2 and 7 are exactly identical with responses of the metasurfaces based on bilayer graphene with $\mu=0.5 \mathrm{eV}$ and $\mu=0.35 \mathrm{eV}$, respectively, that can be achieved by top-gating [57]. As the last point, it is worth investigating the relative absorption bandwidth of the suggested metasurface absorbers that is expressed as the ratio of the bandwidth to the center frequency; i.e., $W_{B}=2 \times \frac{f_{\max }-f_{\min }}{f_{\max }+f_{\min }} \times 100 \%$, where $f_{\max }$ and $f_{\max }$ denote the maximum and minimum frequencies in the nearly perfect absorption region with $A>90 \%$. For the broadband absorbers, the relative absorption bandwidth is expected to be $>50 \%$. For the solid black curve shown in Fig. 2(b), the calculated value of the absorption bandwidth is almost 58\%, and for the solid black curve in Fig. 7(b), we have $W_{B} \approx 72 \%$.

\section{CONCLUSION}

In conclusion, we have designed and analytically verified a novel and practically simplified graphene-based MPA that is capable of absorbing $\mathrm{THz}$ radiation within a $3 \mathrm{THz}$ absorbing window. The broadband MPA, which comprises a periodic array of graphene patches with two different geometries on a metalbacked $\mathrm{SiO}_{2}$ layer and supports propagating surface plasmon excitations in the broadband region, exhibits polarizationintensive and omnidirectional absorption characteristics. Due to the tunable nature of graphene, it is possible to actively tune the absorption spectrum of the MPA from a resonant response to a broadband one and vice versa. Moreover, it is also possible to tune the spectral location of the absorption bandwidth by modifying the chemical potential of graphene. In an alternative approach, the absorption of the MPA can also be passively modified by adjusting the dimensions of the patches or thickness of the $\mathrm{SiO}_{2}$ spacing layer. Due to its simplified and novel design, it is expected that the proposed graphene-based MPA will find potential interest in $\mathrm{THz}$ spectroscopy and communications.

Funding. Türkiye Bilimsel ve Teknolojik Araştirma Kurumu (TUBITAK) (120E422).

Disclosures. The authors declare no conflicts of interest.

Data Availability. The data underlying the results presented in this paper are not publicly available at this time but may be obtained from the authors upon reasonable request.

\section{REFERENCES}

1. N. Yu, P. Genevet, M. A. Kats, F. Aieta, J.-P. Tetienne, F. Capasso, and Z. Gaburro, "Light propagation with phase discontinuities: generalized laws of reflection and refraction," Science 334, 333-337 (2011).

2. F. Aieta, P. Genevet, N. Yu, M. A. Kats, Z. Gaburro, and F. Capasso, "Out-of-plane reflection and refraction of light by anisotropic optical antenna metasurfaces with phase discontinuities," Nano Lett. 12, 1702-1706 (2012).

3. F. Aieta, P. Genevet, M. A. Kats, N. Yu, R. Blanchard, Z. Gaburro, and F. Capasso, "Aberration-free ultrathin flat lenses and axicons at telecom wavelengths based on plasmonic metasurfaces," Nano Lett. 12, 4932-4936 (2012). 
4. M. Khorasaninejad, W. T. Chen, R. C. Devlin, J. Oh, A. Y. Zhu, and F. Capasso, "Metalenses at visible wavelengths: diffraction-limited focusing and subwavelength resolution imaging," Science $\mathbf{3 5 2}$, 1190-1194 (2016).

5. Z. Ma, S. M. Hanham, P. Albella, B. Ng, H. T. Lu, Y. Gong, S. A. Maier, and M. Hong, "Terahertz all-dielectric magnetic mirror metasurfaces," ACS Photon. 3, 1010-1018 (2016).

6. V. Erçaglar, V. Erturk, A. Ghobadi, D. U. Yildirim, and E. Ozbay, "Numerical analysis of a thermally tunable spectrally selective absorber enabled by an all-dielectric metamirror," Opt. Lett. 45, 6174-6177 (2020).

7. O. V. Polischuk, V. S. Melnikova, and V. V. Popov, "Giant crosspolarization conversion of terahertz radiation by plasmons in an active graphene metasurface," Appl. Phys. Lett. 109, 131101 (2016).

8. T. Li, X. Hu, H. Chen, C. Zhao, Y. Xu, X. Wei, and G. Song, "Metallic metasurfaces for high efficient polarization conversion control in transmission mode," Opt. Express 25, 23597-23604 (2017).

9. S. Sun, Z. Zhou, C. Zhang, Y. Gao, Z. Duan, S. Xiao, and Q. Song, "Alldielectric full-color printing with $\mathrm{TiO}_{2}$ metasurfaces," ACS Nano 11, 4445-4452 (2017).

10. G. Zheng, H. Mühlenbernd, M. Kenney, G. Li, T. Zentgraf, and S. Zhang, "Metasurface holograms reaching $80 \%$ efficiency," Nat. Nanotechnol. 10, 308 (2015).

11. Q. Jiang, G. Jin, and L. Cao, "When metasurface meets hologram: principle and advances," Adv. Opt. Photon. 11, 518-576 (2019).

12. X. Wang, Z. Nie, Y. Liang, J. Wang, T. Li, and B. Jia, "Recent advances on optical vortex generation," Nanophotonics 7, 1533 (2018).

13. Y. Zhang, X. Yang, and J. Gao, "Orbital angular momentum transformation of optical vortex with aluminum metasurfaces," Sci. Rep. 9, 9133 (2019).

14. M. Wei, Q. Xu, Q. Wang, X. Zhang, Y. Li, J. Gu, Z. Tian, X. Zhang, J. Han, and W. Zhang, "Broadband non-polarizing terahertz beam splitters with variable split ratio," Appl. Phys. Lett. 111, 071101 (2017).

15. F. Ding, R. Deshpande, C. Meng, and S. I. Bozhevolnyi, "Metasurface-enabled broadband beam splitters integrated with quarter-wave plate functionality," Nanoscale 12, 14106 (2020).

16. N. I. Landy, S. Sajuyigbe, J. Mock, D. Smith, and W. Padilla, "Perfect metamaterial absorber," Phys. Rev. Lett. 100, 207402 (2008).

17. N. Mattiucci, M. J. Bloemer, N. Aközbek, and G. D’Aguanno, "Impedance matched thin metamaterials make metals absorbing," Sci. Rep. 3, 3203 (2013).

18. H. Hajian, A. Ghobadi, B. Butun, and E. Ozbay, "Active metamaterial nearly perfect light absorbers: a review," J. Opt. Soc. Am. B 36, F131-F143 (2019).

19. K. B. Alici, F. Bilotti, L. Vegni, and E. Ozbay, "Experimental verification of metamaterial based subwavelength microwave absorbers," J. Appl. Phys. 108, 083113 (2010).

20. J. W. Park, P. V. Tuong, J. Y. Rhee, K. W. Kim, W. H. Jang, E. H. Choi, L. Y. Chen, and Y. Lee, "Multi-band metamaterial absorber based on the arrangement of donut-type resonators," Opt. Express 21, 96919702 (2013).

21. H. T. Chen, "Interference theory of metamaterial perfect absorbers," Opt. Express 20, 7165-7172 (2012).

22. Y. He, Q. Wu, and S. Yan, "Multi-band terahertz absorber at $0.1-1 \mathrm{THz}$ frequency based on ultra-thin metamaterial," Plasmonics 14, 13031310 (2019).

23. S. Barzegar-Parizi and A. Khavasi, "Designing dual-band absorbers by graphene/metallic metasurfaces," IEEE J. Quantum Electron. 55, 1-8 (2019).

24. G. Yao, F. Ling, J. Yue, C. Luo, J. Ji, and J. Yao, "Dual-band tunable perfect metamaterial absorber in the THz range," Opt. Express 24, 1518-1527 (2016).

25. N. Hu, F. Wu, L. Bian, H. Liu, and P. Liu, "Dual broadband absorber based on graphene metamaterial in the terahertz range," Opt. Mater. Express 8, 3899-13894 (2018).

26. K. Aydin, V. E. Ferry, R. M. Briggs, and H. A. Atwater, "Broadband polarization-independent resonant light absorption using ultrathin plasmonic super absorbers," Nat. Commun. 2, 517 (2011).

27. M. G. Nielsen, A. Pors, O. Albrektsen, and S. I. Bozhevolnyi, "Efficient absorption of visible radiation by gap plasmon resonators," Opt. Express 20, 13311-13319 (2012).
28. F. Ding, J. Dai, Y. Chen, J. Zhu, Y. Jin, and S. I. Bozhevolnyi, "Broadband near-infrared metamaterial absorbers utilizing highly lossy metals," Sci. Rep. 6, 39445 (2016).

29. X. Chen, J. H. Wu, C. R. Liu, and P. Cao, "Ultra-broadband wide angle perfect absorber in the visible regime through a thin grating insulatormetal structure," J. Opt. Soc. Am. B 36, 153-161 (2019).

30. K. Gorgulu, A. Gok, M. Yilmaz, K. Topalli, N. Bıyıklı, and A. K. Okyay, "All-silicon ultra-broadband infrared light absorbers," Sci. Rep. 6, 38589 (2016).

31. L. Ye, Y. Chen, G. Cai, N. Liu, J. Zhu, Z. Song, and Q. H. Liu, "Broadband absorber with periodically sinusoidally-patterned graphene layer in terahertz range," Opt. Express 25, 11223-11232 (2017).

32. C. Liu, L. Qi, and X. Zhang, "Broadband graphene-based metamaterial absorbers," AIP Adv. 8, 015301 (2018).

33. P. Kumar, A. Lakhtakia, and P. K. Jain, "Graphene pixel-based polarization-insensitive metasurface for almost perfect and wideband terahertz absorption,” J. Opt. Soc. Am. B 36, F84-F88 (2019).

34. L. Qi, C. Liu, and S. M. A. Shah, "A broad dual-band switchable graphene-based terahertz metamaterial absorber," Carbon 153, 179 (2019).

35. Z. Liu, L. Guo, and Q. Zhang, "A simple and efficient method for designing broadband terahertz absorber based on singular graphene metasurface," Nanomaterials 9, 1351 (2019).

36. T. Wang, Y. Zhang, H. Zhang, and M. Cao, "Dual-controlled switchable broadband terahertz absorber based on a graphene-vanadium dioxide metamaterial," Opt. Mater. Express 10, 369-386 (2020).

37. S. Quader, M. R. Akram, F. Xiao, and W. Zhu, "Graphene based ultrabroadband terahertz metamaterial absorber with dual-band tunability," J. Opt. 22, 095104 (2020).

38. A. Fardoost, F. Ghaedi Vanani, A. Amirhosseini, and R. Safian, "Design of a multilayer graphene-based ultrawideband terahertz absorber," IEEE Trans. Nanotechnol. 16, 68-74 (2017).

39. S. Biabanifard, M. Biabanifard, S. Asgari, S. Asadi, and C. E. Mustapha, "Tunable ultra-wideband terahertz absorber based on graphene disks and ribbons," Opt. Commun. 427, 418-425 (2018).

40. L. Liu, W. Liu, and Z. Song, "Ultra-broadband terahertz absorber based on a multilayer graphene metamaterial," J. Appl. Phys. 128, 093104 (2020).

41. F. Ding, Y. Cui, X. Ge, Y. Jin, and S. He, "Ultra-broadband microwave metamaterial absorber," Appl. Phys. Lett. 100, 103506 (2012).

42. R. Badugu and J. R. Lakowicz, "Tamm state-coupled emission: effect of probe location and emission wavelength," J. Phys. Chem. C 118, 21558-21571 (2014).

43. M. G. Nielsen, D. K. Gramotnev, A. Pors, O. Albrektsen, and S. I. Bozhevolnyi, "Continuous layer gap plasmon resonators," Opt. Express 19, 19310-19322 (2011).

44. X. Liu, T. Tyler, T. Starr, A. F. Starr, N. M. Jokerst, and W. J. Padilla, "Taming the blackbody with infrared metamaterials as selective thermal emitters," Phys. Rev. Lett. 107, 045901 (2011).

45. V. Erçağlar, H. Hajian, and E. Ozbay, " $\mathrm{VO}_{2}$-graphene-integrated hBNbased metasurface for bi-tunable phonon-induced transparency and nearly perfect resonant absorption," J. Phys. D 54, 245101 (2021).

46. A. Gong, Y. Qiu, X. Chen, Z. Zhao, L. Xia, and Y. Shao, "Biomedical applications of terahertz technology," Appl. Spectrosc. Rev. 55, 418 (2020).

47. C. Baker, W. R. Tribe, B. E. Cole, and M. C. Kemp, "The role of terahertz technology in security applications and people screening," in Optical Terahertz Science and Technology (OTST) (Optical Society of America, 2005), paper TuD2.

48. J. Federici and L. Moeller, "Review of terahertz and subterahertz wireless communications," J. Appl. Phys. 107, 111101 (2010).

49. X. Ropagnol, M. Matoba, J. E. Nkeck, F. Blanchard, E. Isgandarov, J. Yumoto, and T. Ozaki, "Efficient terahertz generation and detection in cadmium telluride using ultrafast ytterbium laser," Appl. Phys. Lett. 117, 181101 (2020).

50. Z. Zhu, K. Cai, J. Deng, V. P. K. Miriyala, H. Yang, X. Fong, and G. Liang, "Electrical generation and detection of terahertz signal based on spin-wave emission from ferrimagnets," Phys. Rev. Appl. 13, 034040 (2020). 
51. H. Chen, W. J. Padilla, J. M. O. Zide, A. C. Gossard, A. J. Taylor, and R. D. Averitt, "Active terahertz metamaterial devices," Nature 444, 597-600 (2006).

52. S. Wang, L. Kang, and D. H. Werner, "Hybrid resonators and highly tunable terahertz metamaterials enabled by vanadium dioxide $\left(\mathrm{VO}_{2}\right)$," Sci. Rep. 7, 4326 (2017).

53. D. Chen, J. Yang, J. Huang, W. Bai, J. Zhang, Z. Zhang, S. Xu, and W. Xie, "The novel graphene metasurfaces based on split-ring resonators for tunable polarization switching and beam steering at terahertz frequencies," Carbon 154, 350-356 (2019).
54. Lumerical Inc., https://www.lumerical.com/products/.

55. L. A. Falkovsky, "Optical properties of graphene," J. Phys. Conf. Ser. 129, 012004 (2008).

56. E. D. Palik, Handbook of Optical Constants of Solids (Elsevier, 1988). 57. Z. Fang, S. Thongrattanasiri, A. Schlather, Z. Liu, L. Ma, Y. Wang, P. M. Ajayan, P. Nordlander, N. J. Halas, and F. J. García de Abajo, "Gated tunability and hybridization of localized plasmons in nanostructured grapheme," ACS Nano 7, 2388 (2013). 